\title{
Can the accurate epidemiological study help to search the etiology of Kawasaki disease?
}

\author{
Ji Whan Han, MD, PhD \\ Department of Pediatrics, Yeouido St. Mary's Hospital, The Catholic University of Korea, Seoul, Korea
}

The first case of Kawasaki disease was reported by Dr. Tomisaku Kawasaki in 1961. He published the data of 50 patients with Kawasaki disease were in the Japanese journal, Arerugi and the American Journal of Pediatrics in 1967 and 1974, respectively. ${ }^{1)}$ Thereafter, many Japanese and North American pediatricians were able to recognize Kawasaki disease.

The etiology of Kawasaki disease remains undetermined despite many trials. Epidemiological studies of a disease can provide a new understanding or insight of the pathophysiology or risk factors of Kawasaki disease. The Japanese nationwide epidemiological surveys on Kawasaki disease have been performed biennially, and more than 300,000 patients who had Kawasaki disease as young children have been registered in the Japanese Department of Health, Labour, and Welfare since 1970.2) Otherwise, nationwide epidemiological surveys on Kawasaki disease have been performed triennially in South Korea and Taiwan since 1994 and 1996, respectively. 3,4$)$

The most useful method for determining the epidemiology of Kawasaki disease is the nationwide survey with well-organized questionnaires like the ones used in South Korea and Japan. ${ }^{3,5}$ This can provide us with data pertaining to incidences, laboratory findings, coronary artery complication rates, etc. Therefore, we can possibly infer the pathophysiology of and risk factors for Kawasaki disease by using these data. Two most important factors for obtaining true data from nationwide surveys are the exact diagnosis of Kawasaki disease and active responses to the surveys by pediatricians who care for affected patients. However, Kawasaki disease can be erroneously diagnosed by health-care providers (general practitioners and pediatricians) and parents of patients with Kawasaki disease in many countries, including South Korea. ${ }^{6}$ The over- and underdiagnosis of Kawasaki disease can significantly influence epidemiological survey data accuracy. Accurate epidemiological surveys on Kawasaki disease are necessary worldwide to increase the understanding and proper management of Kawasaki disease.

In South Korea and Taiwan, National Health Insurance Service (NHIS) data are also utilized for epidemiological surveys on Kawasaki disease. ${ }^{3,4)}$ This is noteworthy because the NHIS programs of South Korea and Taiwan cover more than 99\% of the population due to the government's compulsory policy. However, NHIS data can be inaccurate. For instance, the number of patients with Kawasaki disease who did not receive intravenous immunoglobulin could be excluded from the NHIS data. ${ }^{3)}$ In the United States (US), the passive national surveillance system reports to the Centers for Disease Control and Prevention, while an inpatient database of children and nationwide inpatient samples has been used for epidemiological surveys since the mid-1970s. ${ }^{7)}$

The incidence rate of Kawasaki disease is much higher in Northeast Asia than in North America and Europe and continues to increase steadily. The latest incidence rates of Kawasaki disease were 308 in Japan (2014), 194.7 in South Korea (2014), 111.6 in Beijing (2014), 71.9 in Shanghai (2014), and 82.8 per 100,000 children aged below 5-years in Taiwan (2010). ${ }^{6}$ In North America, the incidence rates of Kawasaki disease were 19.6 in Canada (2014) and 19.1 per 100,000 children aged below 5 years in the US (2015).6 Interestingly, outside Northeast Asian countries, the incidence of Kawasaki disease has not changed significantly since $1973,{ }^{5)}$ but this might be because several cases go unreported or undiagnosed. In North America and Europe, most patients with Kawasaki disease are Asian. In Hawaii, the incidence rate of Kawasaki disease in Japanese Americans is as high as that reported in Japan. These data indicate that genetic susceptibility is closely related to the occurrence of Kawasaki disease. $^{5)}$

The guideline for the diagnosis of Kawasaki disease has been revised since the first Japanese guideline was published in 1984. ${ }^{8}$ The American Heart Association's guideline in 2017 newly recommends that Kawasaki disease can be diagnosed in the presence of a 4-day fever together with at least 4 other principal clinical features of Kawasaki disease, although experienced clinicians who have treated many patients with Kawasaki disease be able to establish the diagnosis with a 3-day fever in rare cases.9) These recommendations can empower the specialist to treat patients with Kawasaki disease as soon as possible, thereby reducing coronary artery complications.

Corresponding author: Ji Whan Han, MD, PhD, Department of Pediatrics, Yeouido St. Mary's Hospital, The Catholic University of Korea, 10, 63-ro, Yeongdeungpo-gu, Seoul 07345, Korea

凶E-mail: han59@catholic.ac.kr, hanji59@gmail.com, https://orcid.org/0000-0002-3024-0609

Received: 22 April, 2019, Revised: 28 June, 2019, Accepted: 31 July, 2019

This is an open-access article distributed under the terms of the Creative Commons Attribution Non-Commercial License (http://creativecommons.org/licenses/bync/4.0/) which permits unrestricted non-commercial use, distribution, and reproduction in any medium, provided the original work is properly cited.

Copyright (c) 2020 by The Korean Pediatric Society 
Two interesting findings from the epidemiological surveys on Kawasaki disease are seasonal peaks of incidence and geographical outbreak tendencies, which imply that some infectious agents may play roles in the pathogenesis of Kawasaki disease. It is postulated that some infectious agents may stimulate the immune system and induce Kawasaki disease in susceptible patients. ${ }^{9}$ The meticulous surveillance of patients with Kawasaki disease by health care providers can enable the quick diagnosis and treatment of Kawasaki disease, thereby preventing coronary artery complications, such as giant aneurysms, and death. $\left.{ }^{6}\right)$

The important factors for collecting exact epidemiological data are definite diagnosis, accurate number of patients with Kawasaki disease, and active participation of clinicians in epidemiological surveys. Among the tools of nationwide surveys on Kawasaki disease, questionnaire data are more accurate than NHIS data. In conclusion, the combination of nationwide epidemiological surveys and use of NHIS data on Kawasaki disease will efficiently achieve an accurate epidemiological study of Kawasaki disease, which would help identify the etiology of Kawasaki disease.

\section{Conflicts of interest}

No potential conflict of interest relevant to this article was reported.
See the article "Reality of Kawasaki disease epidemiology" in Volume 62 on page 292.

\section{References}

1. Kawasaki T, Kosaki F, Okawa S, Shigematsu I, Yanagawa H. A new infantile acute febrile mucocutaneous lymph node syndrome (MLNS) prevailing in Japan. Pediatrics 1974;54:271-6.

2. Nakamura Y. Kawasaki disease: epidemiology and the lessons from it. Int $J$ Rheum Dis 2018;21:16-9.

3. Kim GB, Park S, Eun LY, Han JW, Lee SY, Yoon KL, et al. Epidemiology and clinical features of Kawasaki disease in South Korea, 2012-2014. Pediatr Infect Dis J 2017;36:482-5.

4. Lin MC, Lai MS, Jan SL, Fu YC. Epidemiologic features of Kawasaki disease in acute stages in Taiwan, 1997-2010: effect of different case definitions in claims data analysis. J Chin Med Assoc 2015;78:121-6.

5. Makino N, Nakamura Y, Yashiro M, Sano T, Ae R, Kosami K, et al. Epidemiological observations of Kawasaki disease in Japan, 2013-2014. Pediatr Int 2018;60:581-7.

6. Kim GB. Reality of Kawasaki disease epidemiology. Korean J Pediatr 2019;62:292-6.

7. Uehara R, Belay ED. Epidemiology of Kawasaki disease in Asia, Europe, and the United States. J Epidemiol 2012;22:79-85.

8. Ayusawa M, Sonobe T, Uemura S, Ogawa S, Nakamura Y, Kiyosawa N, et al. Revision of diagnostic guidelines for Kawasaki disease (the 5 th revised edition). Pediatr Int 2005;47:232-4.

9. McCrindle BW, Rowley AH, Newburger JW, Burns JC, Bolger AF, Gewitz $\mathrm{M}$, et al. Diagnosis, treatment, and long-term management of Kawasaki disease: a scientific statement for health professionals from the American Heart Association. Circulation 2017;135:e927-99. 